\begin{tabular}{|c|c|}
\hline Title & A cross sectional exploratory survey on occurrence of triple-whammy prescription pattern in Japan \\
\hline Author(s) & Imai, Shungo; Momo, Kenji; Kashiwagi, Hitoshi; Miyai, T akayuki; Sugawara, Mitsuru; Takekuma, Y oh \\
\hline Citation & $\begin{array}{l}\text { International Journal of Clinical Pharmacy, 42, 1369-1373 } \\
\text { https://doi.org/10.1007/s11096-020-01088-z }\end{array}$ \\
\hline Issue Date & $2020-08-17$ \\
\hline Doc URL & http://hdl.handle.net/2115/82453 \\
\hline Rights & $\begin{array}{l}\text { This is a post-peer-review, pre copy edit version of an article published in International Journal of Clinical Pharmacy. } \\
\text { The final authenticated version is available online at: http://dx.doi.org/10.1007/s11096-020-01088-z. }\end{array}$ \\
\hline Type & article (author version) \\
\hline File Information & WoS_95661_Imai.pdf \\
\hline
\end{tabular}

Instructions for use 


\section{A cross-sectional exploratory survey on occurrence of triple-whammy prescription pattern in Japan}

Shungo Imai ${ }^{1}$, Kenji Momo ${ }^{2}$, Hitoshi Kashiwagi ${ }^{1}$, Takayuki Miyai ${ }^{3}$, Mitsuru Sugawara ${ }^{1,4}$, Yoh Takekuma ${ }^{* 4}$

${ }^{1}$ Faculty of Pharmaceutical Sciences, Hokkaido University, Kita 12-jo Nishi 6-chome, Kita-ku, Sapporo 060-0812, Japan:

${ }^{2}$ Department of Hospital Pharmaceutics, School of Pharmacy, Showa University, 1-5-8 Hatanodai, Shinagawa-ku, Tokyo 142-8555, Japan:

${ }^{3}$ Graduate School of Life Science, Hokkaido University, Kita 10-jo Nishi 8-chome, Kita-ku, Sapporo 0600810, Japan: and

${ }^{4}$ Department of Pharmacy, Hokkaido University Hospital, Kita 14-jo, Nishi 5-chome, Kita-ku, Sapporo 0608648, Japan.

*Corresponding author:

Yoh Takekuma

Department of Pharmacy, Hokkaido University Hospital, Kita 14-jo, Nishi 5-chome, Kita-ku, Sapporo 0608648, Japan.

E-mail: y-kuma@pharm.hokudai.ac.jp

ORCID iD: 0000-0002-4325-0785 


\title{
A cross-sectional exploratory survey on occurrence of triple-whammy prescription pattern in Japan
}

\begin{abstract}
Background: The concurrent use of nonsteroidal anti-inflammatory drugs, renin-angiotensin-aldosterone system blockers, and diuretics, known as a "triple-whammy," is related to the occurrence of acute kidney injury. However, there are few reports regarding the prescription pattern of the triple-whammy.

Objective: To elucidate the patterns of the triple-whammy prescription in Japan.

Methods: A cross-sectional study was performed using a health-insurance-claims database that included Japanese people under 75 years of age, and enrolled outpatients that were prescribed any nonsteroidal anti-inflammatory drugs, renin-angiotensin-aldosterone system blockers, and diuretics between April 2017 and June 2017. As an outcome, the proportion of triple-whammy prescriptions was evaluated. Among the patients who received triple-whammy prescriptions, we evaluated the prevalence of chronic kidney disease and the proportion of prescriptions provided for these three drugs from different clinical departments and institutions.

Results: Overall, 730 of 246,721 (0.3\%) patients received triple-whammy prescriptions. Among these patients, $13.3 \%$ had underlying chronic kidney disease. The proportions of any of the three drug types prescribed by different clinical departments and institutions was $48.2 \%$ and $61.8 \%$, respectively.

Conclusions: We examined the patterns of triple-whammy prescriptions and concluded that pharmacists need to pay attention to triple-whammy prescriptions if the prescriptions are provided by multiple clinical departments or institutions.
\end{abstract}

Keywords Triple-whammy prescriptions, Insurance claims, Acute kidney injury, Japan

\section{Impact of findings on practice statements}

- To avoid acute kidney injury, clinicians and pharmacists should be aware of "triple-whammy" prescriptions (i.e., concomitant use of nonsteroidal anti-inflammatory drugs, renin-angiotensinaldosterone system blockers, and diuretics) because $0.3 \%$ of Japanese patients under 75 years of age who were prescribed any of these drugs received triple-whammy prescriptions.

It should be noted that $13.3 \%$ of patients with triple-whammy prescriptions had underlying chronic kidney disease.

In particular, pharmacists should be aware of triple-whammy if prescriptions are performed by multiple clinical departments or institutions. 


\section{Introduction}

Concurrent use of nonsteroidal anti-inflammatory drugs (NSAIDs), renin-angiotensin-aldosterone system (RAAS) blockers, and diuretics is strongly related to the occurrence of acute kidney injury (AKI); this combination of drugs is called the "triple-whammy" [1-4]. AKI induced by the triple-whammy is caused by a combination of pharmacological activities: (1) NSAIDs lead to renal afferent arteriolar constriction; (2) RAAS blockers cause hemodynamic reduction in the glomerular-filtration rate due to efferent arteriolar dilation, and (3) diuretics lead to hypovolemia [1,3]. Thus, Japanese guidelines recommend that particular attention be paid to prescription of triple-whammy to patients with chronic kidney disease (CKD) because these combinations are likely to induce disease progression [5].

In our clinical-pharmacy-practice experience, triple-whammy prescriptions often occur when the three drug types are prescribed by different clinical departments. For example, NSAIDs may be prescribed by an orthopedic surgery department, whereas RAAS blockers and diuretics may be prescribed by an internal medicine department. However, no reports have assessed the extent to which this occurs.

\section{Aims of the study}

We aimed to elucidate the patterns of the triple-whammy prescription by using a health-insurance-claims database in Japan.

\section{Ethics approval}

This research was reviewed by the Institutional Review Board (IRB) of the Faculty of Pharmaceutical Sciences at Hokkaido University; the IRB waived the requirement for informed consent owing to the anonymous nature of the data (approval number not given).

\section{Method}

\section{Data sources and study population}

This cross-sectional study was performed using JMDC claims database, a Japanese health-insuranceclaims database maintained by JMDC, Inc. (Tokyo, Japan). This database contains monthly claims from medical institutions and pharmacies from January 2005 to June 2017 (as of June 2018), and covers approximately 5.6 million registered individuals (approximately $5 \%$ of Japan population). The study included registered employees of companies and their family members under 75 years of age [6].

Among the registered individuals, we enrolled outpatients who were prescribed any oral NSAIDs, oral RAAS blockers, and oral diuretics between April 2017 and June 2017. In Japan, as a prescription period is usually shorter than 3 months, we observed a 3-month period (i.e., from April 2017 to June 2017) to 
evaluate one prescription cycle. Patients who received occasional prescriptions were detected by the "occasional use" flag in the JMDC claims database and excluded because of the difficulty with calculating the administration period.

\section{Data collection}

Drugs were identified by the Anatomical Therapeutic Chemical (ATC) system: NSAIDs (M01A); RAAS blockers (C09A, C09C, and C09D); and Diuretics (C03). RAAS blockers were defined as angiotensin II receptor blockers (ARB) and angiotensin-converting-enzyme inhibitors (ACE-I) [4]. Clinical departments and institutions were extracted from text codes and institution IDs. Clinical departments registered as "other clinical departments" were classified as "other". The 10 most common NSAIDs, RAAS blockers, and diuretics were chosen for evaluating patient characteristics.

As an underlying disease, CKD was evaluated based on the diagnostic criteria of the International Classification of Diseases, Tenth Revision (ICD-10) codes of N180, N188, N189, and N289. For the ICD10 code of N289, CKD stages 1 and 2 (determined by text code) were assessed as non-CKD.

Additionally, patients receiving dialysis (detected by standard clinical-practice text) were defined as nonCKD. To identify CKD diagnosis, we screened patient data from January 2016 to the study period. Moreover, data on patient age and sex were collected.

\section{Outcomes}

The proportion of triple-whammy prescriptions was evaluated as an endpoint. The procedure for detecting triple-whammy prescriptions was as follows (Online Resource 1): (1) the first prescription of any NSAIDs, RAAS blockers, and diuretics between April 2017 and June 2017 (employed as the denominator); (2) in prescriptions identified in (1), the occurrence of an overlapping-prescription period of the other two drug types prescribed between January 2017 and June 2017 (employed as the numerator). For example, for patients prescribed NSAIDs, prescriptions for RAAS blockers and/or diuretics were examined. Moreover, the prevalence of CKD was evaluated (the definition of CKD is provided in the data-collection statement).

For patients with triple-whammy prescriptions, the information on clinical departments that prescribed these drugs was collected. In addition, we evaluated the proportion of prescriptions of these drugs from different clinical departments and institutions. Moreover, the frequency of prescriptions from a combination of clinical departments was evaluated.

\section{Statistical analyses}

Categorical variables were compared by Pearson's chi-square or Fisher's exact test. As the continuous variable (patient age) was confirmed to have non-normal distribution by the Kolmogorov-Smirnov test, the Mann-Whitney U test was used. To perform multiple comparisons, adjusted $P$-values were calculated 
using Bonferroni corrections. A significant difference was defined as a $P$-value $<0.05$. Statistical analyses were computed by using JMP $14^{\circledR}$ software (SAS Institute, Inc., Cary, NC, USA).

\section{Results}

Of the outpatients prescribed any oral NSAIDs, oral RAAS blockers, and oral diuretics between April 2017 to June 2017 ( $\mathrm{n}=252,702)$, patients who received occasional prescriptions were excluded ( $\mathrm{n}=$ $5,981)$. Overall, 730 of 246,721 ( $0.3 \%)$ patients received triple-whammy prescriptions (Online Resource 2).

The prevalence of CKD was significantly higher in the triple-whammy-prescription group than in patients without triple-whammy prescription $(\mathrm{n}=97,13.3 \%$ and $\mathrm{n}=18,003,7.3 \%$, respectively, $P<$ 0.001). Other details of triple-whammy prescriptions are shown in Online Resource 3 and 4.

Of the clinical departments, internal medicine departments prescribed most of the drugs assessed (Table 1). More NSAIDs than other drugs were prescribed by the orthopedic surgery and otorhinolaryngology departments. The proportion of prescriptions comprising the three drug types by different clinical departments and institutions was $48.2 \%$ and $61.8 \%$, respectively (Figure 1A). All drug types were most often prescribed by internal medicine departments (Figure 1B). The second most frequent combination was NSAIDs prescribed by orthopedic surgery departments and RAAS blockers and diuretics by the internal medicine departments.

\section{Discussion}

We found that $0.3 \%$ of the included patients received triple-whammy prescriptions. Although this proportion was lower than the $0.8 \%$ reported in a single-center study [4], we think our results are reasonable because the JMDC claims database only covers patients under 75 years of age [6]. Elderly people have a high risk of AKI induced by the triple-whammy, and polypharmacy is common $[7,8]$. Therefore, as elderly people are considered more at risk, attention should be paid to triple-whammy prescriptions in these patients.

The risk of AKI is increased in patients who received triple-whammy prescriptions, especially patients with renal impairment [7]. In this study, CKD prevalence was $13.3 \%$ in patients in the triple-whammy prescription group, higher than in patients without triple-whammy prescription (7.3\%). This was likely because of the renoprotective effect of RAAS blockers [9]. Furthermore, patients without triple-whammy prescriptions also included those who received only NSAIDs; therefore, the percentage of patients with CKD was higher in the triple-whammy group. Nevertheless, clinicians and pharmacists should be aware 
that $13.3 \%$ of patients in the triple-whammy prescription group had underlying CKD.

As shown in Figure 1A, the proportion of prescribing any of the three drug types by different clinical departments and institutions was $48.2 \%$ and $61.8 \%$, respectively. The percentage for "institutions" was higher than for "clinical departments" owing to the characteristics of the database. For example, details regarding a department registered as "internal medicine" are unknown (e.g., renal internal medicine or oncology internal medicine was not specified). Thus, although the most common occurrence was "all drugs are prescribed by internal medicine departments" (Figure 1B), this included different institutions and may have involved different types of internal medicine. Despite these problems, noteworthily, RAAS blockers and diuretics were also likely to be prescribed by the same department, and NSAIDs alone are often prescribed by a different department, such as orthopedic surgery (Figure 1B; patterns b, c, and e). Thus, pharmacists should be aware of prescriptions from different clinical departments and institutions.

Our study was limited by the following factors that could not be evaluated: (1) actual use of triplewhammy prescriptions; (2) accuracy of CKD diagnoses (although diagnoses of renal disease were obtained with high specificity [10]); (3) severity of CKD (because most diagnoses were not classified into CKD stages); (4) laboratory data (because the database did not have them); and (5) the latest dataset (because the database only contained data up to June 2017). Thus, further research is required to eliminate these limitations.

\section{Conclusion}

To avoid AKI, clinicians and pharmacists need to assess drug-drug interactions, because $0.3 \%$ of enrolled patients received triple-whammy prescriptions. Noteworthily, $13.3 \%$ of patients with triple-whammy prescription had underlying CKD. Moreover, attention to triple-whammy possibility is required if prescriptions are from multiple clinical departments or institutions.

\section{Acknowledgments}

None.

\section{Funding}

None.

\section{Conflicts of interest}

The authors declare no conflict of interest.

\section{References}


1. Loboz KK, Shenfield GM. Drug combinations and impaired renal function -- the 'triple whammy'. Br J Clin Pharmacol, 2005;59:239-43.

2. Wuarin R, Kakkassery V, Consigli A, Roquelaure D, Papanastasiou A, Schutz JS, et al. Combined topical anti-inflammatory and oral acetazolamide in the treatment of central serous chorioretinopathy. Optom Vis Sci, 2019;96:500-6.

3. Lapi F, Azoulay L, Yin H, Nessim SJ, Suissa S. Concurrent use of diuretics, angiotensin converting enzyme inhibitors, and angiotensin receptor blockers with non-steroidal anti-inflammatory drugs and risk of acute kidney injury: nested case-control study. BMJ, 2013;346:e8525.

4. Kunitsu Y, Isono T, Hira D, Terada T. Chronic effects on kidney function by "triple whammy" (combination of renin and angiotensin type inhibitor, diuretic drug and nonsteroidal antiinflammatory drug). Yakugaku Zasshi, 2019;139:1457-62.

5. Japanese Society of Nephrology. Essential points from Evidence-based Clinical Practice Guidelines for Chronic Kidney Disease 2018. Clin Exp Nephrol, 2019;23:1-15.

6. Japan Medical Data Center [Cited 28 Feb 2020]. https://www.jmdc.co.jp/en/.

7. Dreischulte T, Morales DR, Bell S, Guthrie B. Combined use of nonsteroidal anti-inflammatory drugs with diuretics and/or renin-angiotensin system inhibitors in the community increases the risk of acute kidney injury. Kidney Int, 2015;88:396-403.

8. Fujie K, Kamei R, Araki R, Hashimoto K. Prescription of potentially inappropriate medications in elderly outpatients: a survey using 2015 Japanese Guidelines. Int J Clin Pharm. 2020; doi: 10.1007/s11096-020-00967-9. [Epub ahead of print]

9. Abramowicz D, Cochat P, Claas FH, Heemann U, Pascual J, Dudley C, et al. European Renal Best Practice Guideline on kidney donor and recipient evaluation and perioperative care. Nephrol Dial Transplant, 2015;30:1790-7.

10. Yamana H, Moriwaki M, Horiguchi H, Kodan M, Fushimi K, Yasunaga H. Validity of diagnoses, procedures, and laboratory data in Japanese administrative data. J Epidemiol. 2017;27:476-82. 
Table 1. Clinical departments prescribing NSAIDs, RAAS blockers, and diuretics in the triplewhammy-prescriptions group

\begin{tabular}{llll}
\hline Description & $\begin{array}{l}\text { NSAIDs } \\
(\mathrm{n}=730)\end{array}$ & $\begin{array}{l}\text { RAAS blockers } \\
(\mathrm{n}=730)\end{array}$ & $\begin{array}{l}\text { Diuretics } \\
(\mathrm{n}=730)\end{array}$ \\
\hline Internal Medicine, $\mathrm{n}(\%)$ & $358(49.0)^{*}, \dagger$ & $588(80.6)$ & $579(79.3)$ \\
Orthopedic Surgery, $\mathrm{n}(\%)$ & $228(31.2)^{* \dagger} \dagger$ & $9(1.2)$ & $9(1.2)$ \\
General Surgery, $\mathrm{n}(\%)$ & $29(4.0)$ & $19(2.6)$ & $19(2.6)$ \\
Cardiology, $\mathrm{n}(\%)$ & $2(0.3)^{*}$, & $20(2.7)$ & $21(2.9)$ \\
Gastroenterology, $(\%)$ & $11(1.5)$ & $12(1.6)$ & $12(1.6)$ \\
Otorhinolaryngology, $\mathrm{n}(\%)$ & $26(3.6)^{*}, \dagger$ & $0(0.0)$ & $5(0.7)$ \\
Neurosurgery, $\mathrm{n}(\%)$ & $6(0.8)$ & $12(1.6)$ & $12(1.6)$ \\
Respiratory Medicine, $\mathrm{n}(\%)$ & $4(0.5)$ & $9(1.2)$ & $10(1.4)$ \\
Urology, $\mathrm{n}(\%)$ & $5(0.7)$ & $4(0.5)$ & $5(0.7)$ \\
Obstetrics and gynecology, $\mathrm{n}(\%)$ & $4(0.6)$ & $5(0.7)$ & $4(0.5)$ \\
Other Clinical Departments, $\mathrm{n}(\%)$ & $57(7.8)$ & $52(7.1)$ & $54(7.4)$ \\
\hline
\end{tabular}

$* P$ values $\leq 0.05$ versus RAAS blockers, $\dagger P$ values $\leq 0.05$ versus Diuretics; $P$ values were adjusted using Bonferroni correction and values of $\leq 0.05$ were considered statistically significant. NSAIDs: nonsteroidal anti-inflammatory drugs, RAAS: renin-angiotensin-aldosterone system. 


\section{Figure legends}

Figure 1. Different clinical departments and combinations of clinical departments that prescribed triplewhammy prescriptions.

NSAIDs: nonsteroidal anti-inflammatory drugs, RAAS: renin-angiotensin-aldosterone system. 
A. Proportion of triple-whammy prescriptions from different clinical departments and institutions

80

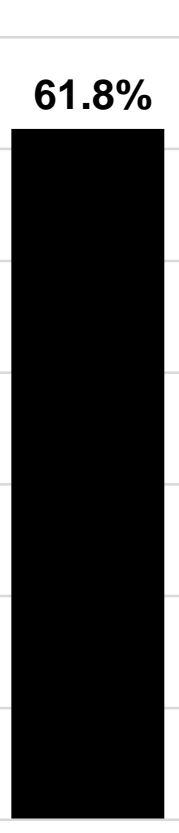

1

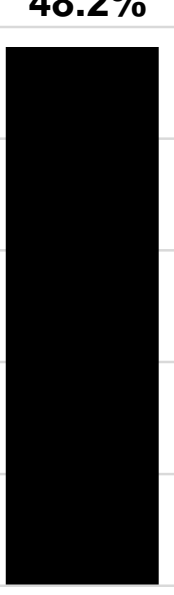

2

$(n=730)$
B. Top five most frequent combinations of departments that prescribed triple-whammy prescriptions

80

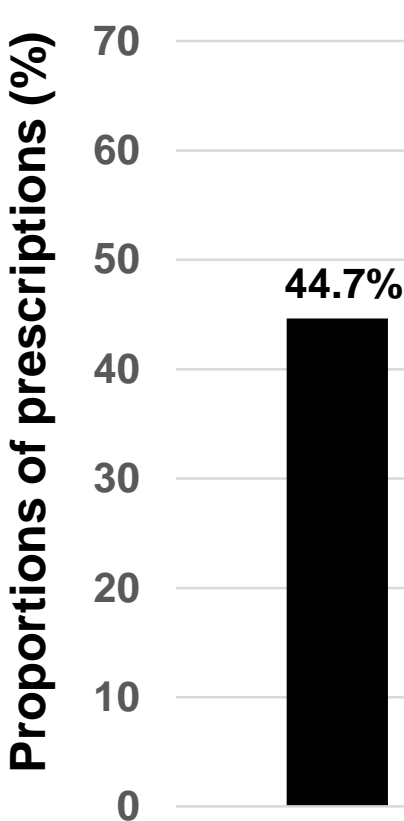

a

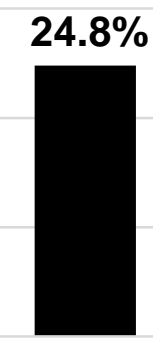

b

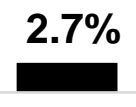

C

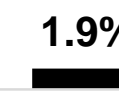

d
$1.6 \%$

e $(n=730)$

\begin{tabular}{ccccc}
\hline \multirow{2}{*}{ Patterns } & $\mathbf{n}$ & \multicolumn{3}{c}{ Clinical departments of prescriptions } \\
\cline { 3 - 5 } & & NSAIDs & RAAS blockers & Diuretics \\
\hline a & 326 & Internal Medicine & Internal Medicine & Internal Medicine \\
b & 181 & Orthopedic Surgery & Internal Medicine & Internal Medicine \\
C & 20 & Otorhinolaryngology & Internal Medicine & Internal Medicine \\
d & 14 & General Surgery & General Surgery & General Surgery \\
e & 12 & Orthopedic Surgery & Cardiology & Cardiology \\
\hline
\end{tabular}


Supplementary_material_1

A cross-sectional exploratory survey on occurrence of triple-whammy prescription pattern in Japan International Journal of Clinical Pharmacy

Shungo Imai, Kenji Momo, Hitoshi Kashiwagi, Takayuki Miyai, Mitsuru Sugawara, Yoh Takekuma

Correspondence to Yoh Takekuma, Department of Pharmacy, Hokkaido University Hospital (email: ykuma@pharm.hokudai.ac.jp)

\section{Online Resource 1}

Figure S1. The procedure for detection of "triple-whammy" prescriptions

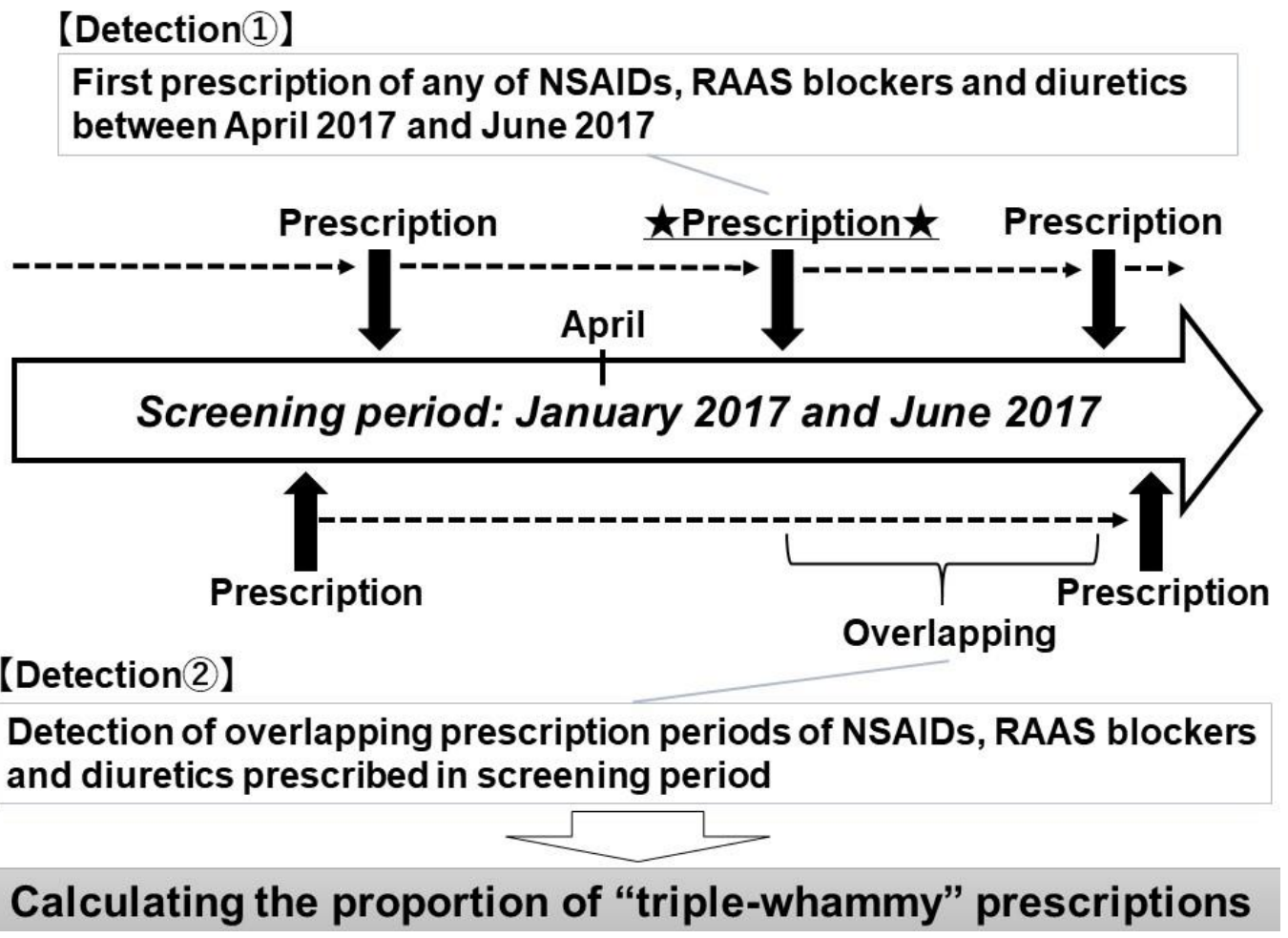

NSAIDs: nonsteroidal anti-inflammatory drugs, RAAS: renin-angiotensin-aldosterone system. 
Supplementary_material_2

A cross-sectional exploratory survey on occurrence of triple-whammy prescription pattern in Japan International Journal of Clinical Pharmacy

Shungo Imai, Kenji Momo, Hitoshi Kashiwagi, Takayuki Miyai, Mitsuru Sugawara, Yoh Takekuma

Correspondence to Yoh Takekuma, Department of Pharmacy, Hokkaido University Hospital (email: ykuma@pharm.hokudai.ac.jp)

\section{Online Resource 2}

Figure S2. Flowchart of patients enrolled in this study and triple-whammy prescription status

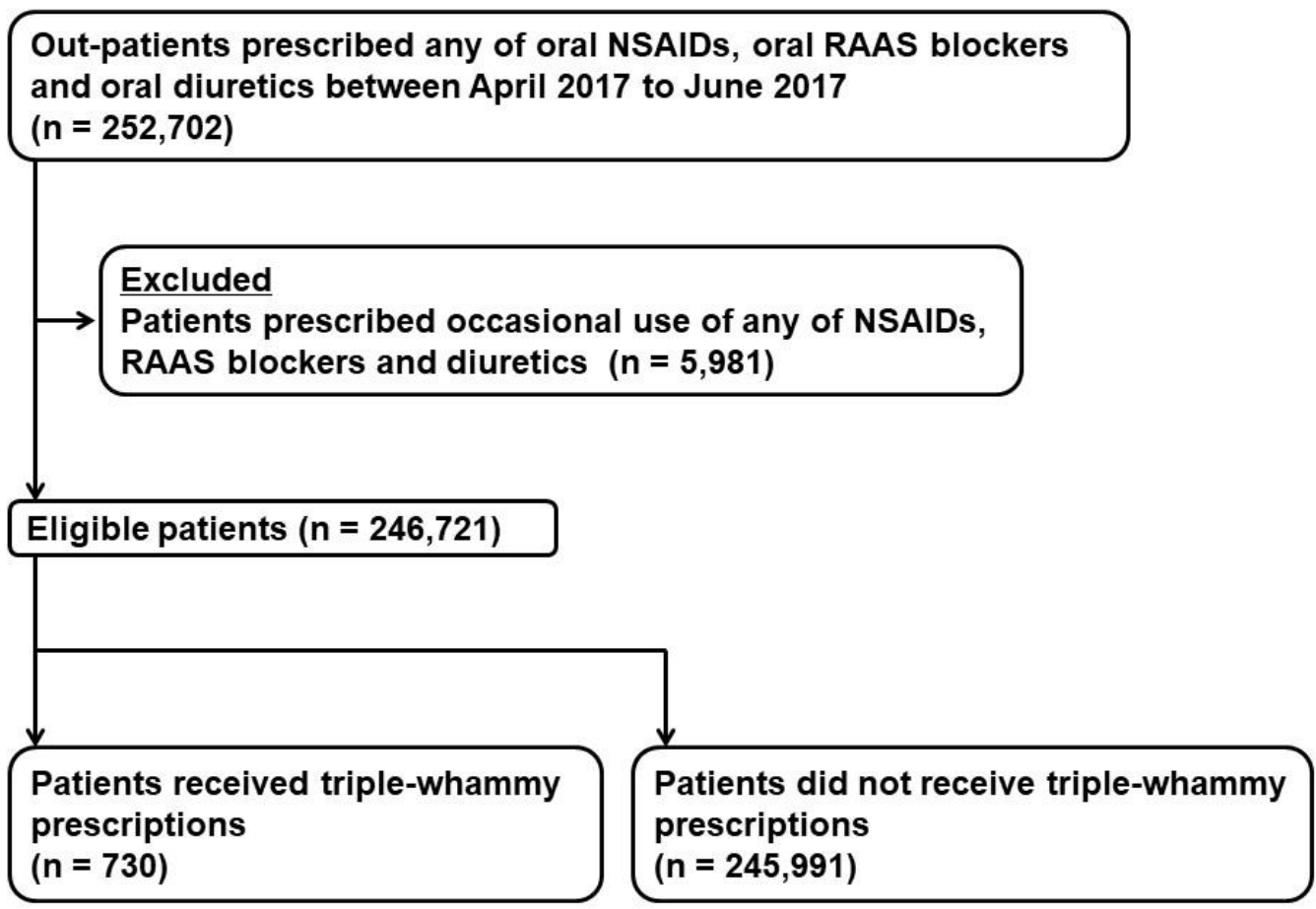

NSAIDs: nonsteroidal anti-inflammatory drugs, RAAS: renin-angiotensin-aldosterone system. 
Supplementary_material_3

A cross-sectional exploratory survey on occurrence of triple-whammy prescription pattern in Japan International Journal of Clinical Pharmacy

Shungo Imai, Kenji Momo, Hitoshi Kashiwagi, Takayuki Miyai, Mitsuru Sugawara, Yoh Takekuma

Correspondence to Yoh Takekuma, Department of Pharmacy, Hokkaido University Hospital (email: ykuma@pharm.hokudai.ac.jp)

\section{Online Resource 3}

Table S1. Comparison of characteristics of patients with or without triple-whammy prescriptions With triple-whammy Without triple-whammy

\begin{tabular}{lccc} 
Description & $\begin{array}{c}\text { prescriptions } \\
(\mathrm{n}=730)\end{array}$ & $\begin{array}{c}\text { prescriptions } \\
(\mathrm{n}=245,991)\end{array}$ & $P$-value \\
\hline Age (years), median (range) & $57(4-74)$ & $49(0-74)$ & $<0.001^{\text {a) } *}$ \\
Sex (male), $\mathrm{n}(\%)$ & $472(64.7)$ & $151,657(61.7)$ & $0.095^{\text {b) }}$ \\
Sex (female), n (\%) & $258(35.3)$ & $94,334(38.3)$ & \\
Prevalence of CKD, n (\%) & $97(13.3)$ & $18,003(7.3)$ & $<0.001^{\text {b) } *}$ \\
\hline
\end{tabular}

a) Mann-Whitney U test, b) Chi-squared test. ${ }^{*} P$ values $\leq 0.05$ were considered statistically significant. CKD: chronic kidney disease. 
Supplementary_material_4

A cross-sectional exploratory survey on occurrence of triple-whammy prescription pattern in Japan International Journal of Clinical Pharmacy

Shungo Imai, Kenji Momo, Hitoshi Kashiwagi, Takayuki Miyai, Mitsuru Sugawara, Yoh Takekuma

Correspondence to Yoh Takekuma, Department of Pharmacy, Hokkaido University Hospital (email: ykuma@pharm.hokudai.ac.jp)

\section{Online Resource 4}

Table S2. Details of oral NSAIDs, oral RAAS blockers, and oral diuretics in the triple-whammy prescriptions group

\begin{tabular}{|c|c|}
\hline Description & Drugs \\
\hline NSAIDs (n) & $\begin{array}{l}\text { Loxoprofen sodium hydrate (444), Celecoxib (119), Diclofenac sodium } \\
\text { (47), Ibuprofen (21), Etodolac (20), Lornoxicam (16), Meloxicam (14), } \\
\text { Bucolome (10), Tiaramide hydrochloride (10), Zaltoprofen (8) }\end{array}$ \\
\hline RAAS blockers (n) & $\begin{array}{l}\text { Olmesartan medoxomil (124), Candesartan cilexetil (124), Telmisartan } \\
\text { (100), Valsartan (98), Azilsartan (96), Irbesartan (69), Losartan } \\
\text { Potassium (47), Enalapril maleate (46), Imidapril hydrochloride (21), } \\
\text { Temocapril hydrochloride (5) }\end{array}$ \\
\hline Diuretics (n) & $\begin{array}{l}\text { Trichlormethiazide (257), Indapamide (132), Furosemide (109), } \\
\text { Spironolactone (89), Eplerenone (72), Azosemide (45), Torasemide } \\
\text { (19), Hydrochlorothiazide (15), Mefruside (10), Isosorbide (7) }\end{array}$ \\
\hline
\end{tabular}

NSAIDs: nonsteroidal anti-inflammatory drugs, RAAS: renin-angiotensin-aldosterone system. There was some overlapping (e.g., multiple NSAIDs prescribed for one patient). The 10 most common drugs of each type are listed. 